\title{
High-velocity runaway stars from three-body encounters
}

\author{
V. V. Gvaramadze, ${ }^{1}$ A. Gualandris ${ }^{2}$ and S. Portegies Zwart ${ }^{3}$ \\ ${ }^{1}$ Sternberg Astronomical Institute, Moscow State University, Universitetskij Pr. 13, \\ Moscow 119992, Russia \\ email: vgvaram@mx.iki.rssi.ru \\ ${ }^{2}$ Center for Computational Relativity and Gravitation, Rochester Institute of Technology, \\ 85 Lomb Memorial Drive, Rochester NY 14623, USA \\ email: alessiag@astro.rit.edu \\ ${ }^{3}$ Astronomical Institute 'Anton Pannekoek' and Section Computational Science, \\ University of Amsterdam, Kruislaan 403, 1098 SJ Amsterdam, the Netherlands \\ email: spz@science.uva.nl
}

\begin{abstract}
We performed numerical simulations of dynamical encounters between hard, massive binaries and a very massive star (VMS; formed through runaway mergers of ordinary stars in the dense core of a young massive star cluster) to explore the hypothesis that this dynamical process could be responsible for the origin of high-velocity $\left(\geqslant 200-400 \mathrm{~km} \mathrm{~s}^{-1}\right)$ early or late B-type stars. We estimated the typical velocities produced in encounters between very tight massive binaries and VMSs (of mass of $\geqslant 200 \mathrm{M}_{\odot}$ ) and found that about $3-4 \%$ of all encounters produce velocities $\geqslant 400 \mathrm{~km} \mathrm{~s}^{-1}$, while in about $2 \%$ of encounters the escapers attain velocities exceeding the Milky Ways's escape velocity. We therefore argue that the origin of high-velocity $\left(\geqslant 200-400 \mathrm{~km} \mathrm{~s}^{-1}\right)$ runaway stars and at least some so-called hypervelocity stars could be associated with dynamical encounters between the tightest massive binaries and VMSs formed in the cores of star clusters. We also simulated dynamical encounters between tight massive binaries and single ordinary $50-100 \mathrm{M}_{\odot}$ stars. We found that from 1 to $\simeq 4 \%$ of these encounters can produce runaway stars with velocities of $\geqslant 300-400 \mathrm{~km} \mathrm{~s}^{-1}$ (typical of the bound population of high-velocity halo B-type stars) and occasionally (in less than $1 \%$ of encounters) produce hypervelocity $\left(\geqslant 700 \mathrm{~km} \mathrm{~s}^{-1}\right.$ ) late B-type escapers.
\end{abstract}

Keywords. stellar dynamics, methods: $N$-body simulations, binaries: general, stars: neutron

\section{Introduction}

The origin of high-velocity runaway stars can be attributed to two basic processes, (i) disruption of a tight, massive binary following the (asymmetric) supernova explosion of one of the binary components (Blaauw 1961) and (ii) dynamical three- or four-body encounters in dense stellar systems (Poveda et al. 1967). In the first process, the maximum velocity attained by runaway stars depends on the magnitude of the kick imparted to the stellar supernova remnant [either a neutron star (NS) or a black hole (BH)] and for reasonable values of this magnitude, the runaway velocity does not exceed $\sim 200 \mathrm{~km} \mathrm{~s}^{-1}$ (e.g., Portegies Zwart 2000; cf. Gvaramadze 2009). In the second process, the ejection velocity could be higher. For example, the maximum velocity that a runaway star can attain in binary-binary encounters is equal to the escape velocity from the surface of the most massive star in the binaries and could be as large as $\sim 1400 \mathrm{~km} \mathrm{~s}^{-1}$ (Leonard 1991).

The recent discovery of the so-called hypervelocity stars (HVSs; e.g., Brown et al. 2005) - ordinary stars moving with velocities exceeding the Milky Way's escape velocityattracted attention to dynamical processes involving the supermassive $\mathrm{BH}$ in the Galactic 
Centre (e.g., Gualandris et al. 2005; Baumgardt et al. 2006). These processes [originally proposed by Hills (1988) and Yu \& Tremaine (2003)] can result in ejection velocities of several $1000 \mathrm{~km} \mathrm{~s}^{-1}$. Similar processes, but acting in the cores of young massive star clusters (YMSCs) and involving dynamical encounters with intermediate-mass $(\sim 100-$ $\left.1000 \mathrm{M}_{\odot}\right)$ BHs (IMBHs), were considered by Gvaramadze et al. (2008) to explain the origin of extremely-high-velocity $\left(\sim 1000 \mathrm{~km} \mathrm{~s}^{-1}\right)$ NSs (e.g., Chatterjee et al. 2005) and HVSs. Gualandris \& Portegies Zwart (2007) proposed that exchange encounters between hard binaries and an IMBH formed in the core of a YMSC in the Large Magellanic Cloud could be responsible for the origin of the HVS HE 0437-5439. Strong support for the possibility that at least some HVSs originate in star clusters rather than in the Galactic Centre comes from the proper-motion measurements of the HVS HD 271791, which constrain the birth place of this star to the outer parts of the Galactic disc (Heber et al. 2008).

\section{High-velocity runaway stars from three-body encounters}

In this paper, we explore the hypothesis (Gvaramadze 2007) that some high-velocity runaway stars could attain their peculiar velocities in the course of strong dynamical encounters between hard, massive binaries and a very massive $\left(\geqslant 100-150 \mathrm{M}_{\odot}\right)$ star (VMS), formed in the core of a YMSC through collisions and mergers of ordinary massive stars (e.g., Portegies Zwart \& McMillan 2002). In this process, one of the binary components is replaced by the VMS, while the second is ejected (a so-called exchange encounter), sometimes with a high velocity. Our goal is to check whether or not this process can produce early B-type stars (the progenitors of NSs) with velocities of $\geqslant 200-400 \mathrm{~km} \mathrm{~s}^{-1}$ (typical of pulsars) and $3-4 \mathrm{M}_{\odot}$ stars with velocities of $\geqslant 300-400 \mathrm{~km} \mathrm{~s}^{-1}$ (typical of some late B-type halo stars).

In our study, we proceed from the similarity between encounters involving a VMS and an IMBH (the latter process is already known to be able to produce high-velocity runaways; Gvaramadze et al. 2008) and the fact that the radii of VMSs are smaller than the tidal radii of the intruders (the massive binaries), so that tidal breakup and ejection can occur before the binary components merge with the VMS (Gvaramadze 2007; Gvaramadze et al. 2009). Our study is motivated by the recent evolutionary models of VMSs (Belkus et al. 2007; Yungelson et al. 2008), which suggest that VMSs can lose most of their mass through copious winds and leave behind IMBHs with masses of $\leqslant 70 \mathrm{M}_{\odot}$, which are not high enough to contribute significantly to the production of high-velocity runaway stars (see Gvaramadze et al. 2008). We therefore explore the possibility that a VMS could produce high-velocity escapers (either early or late B-type stars) before it finishes its life in a supernova and forms a BH. To check this possibility, we performed numerical simulations of three-body exchange encounters using the SIGMA3 package, which is part of the STARLAB software environment (McMillan \& Hut 1996; Portegies Zwart et al. 2001). For a detailed description of the simulations, see Gvaramadze et al. (2009).

First, we focus on exchange encounters producing high-velocity early B-type stars and simulate encounters between a binary consisting of two main-sequence stars with masses $m_{1}=40 \mathrm{M}_{\odot}$ and $m_{2}=8 \mathrm{M}_{\odot}$, and a massive compact body, either a VMS or an ordinary star of mass of $50-100 \mathrm{M}_{\odot}$ (the most massive ordinary stars formed in clusters with mass of $\simeq 10^{3}-10^{4} \mathrm{M}_{\odot}$; e.g., Weidner et al. 2009). To maximize the ejection speed, we assume that the binary system is very tight, e.g., formed by tidal capture. In this case, $a \simeq 3 r_{1} \simeq 0.15 \mathrm{AU}$, where $r_{1}$ is the radius of the primary star (see Gvaramadze et al. 2009). Figure 1 (left panel) shows the velocity distribution for $8 \mathrm{M}_{\odot}$ 

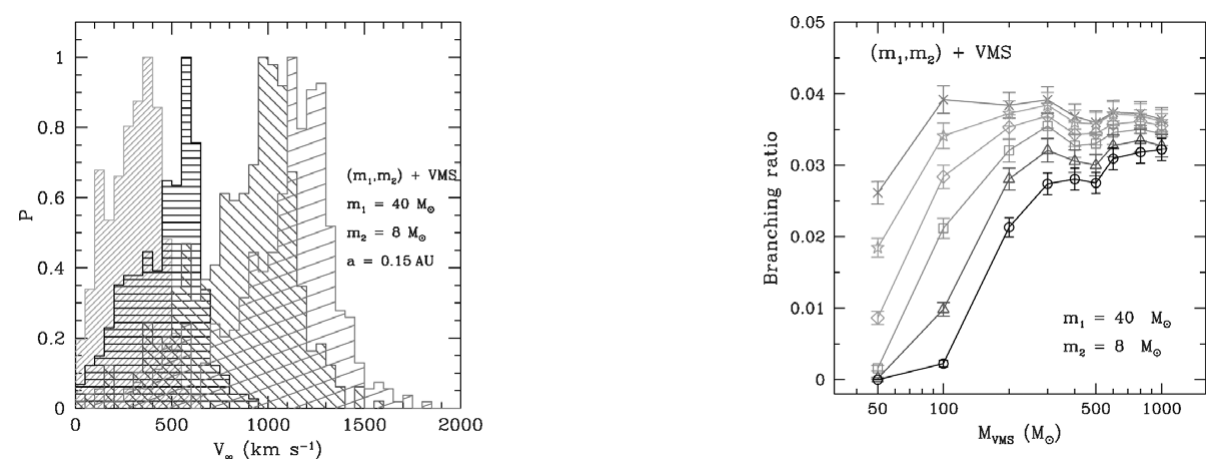

Figure 1. (left) Velocity distributions at infinity for escaping $\left(8 \mathrm{M}_{\odot}\right)$ stars in encounters between a binary consisting of a primary star with mass $m_{1}=40 \mathrm{M}_{\odot}$ and a secondary star with mass $m_{2}=8 \mathrm{M}_{\odot}$, and a single VMS of mass $M_{\mathrm{VMS}}=50 \mathrm{M}_{\odot}, 100 \mathrm{M}_{\odot}, 500 \mathrm{M}_{\odot}$ and $1000 \mathrm{M}_{\odot}$ (from left to right). The binary semi-major axis is $a=0.15 \mathrm{AU}$. (right) Probability of exchange encounters between a $(40,8) \mathrm{M}_{\odot}$ binary (with $a=0.15 \mathrm{AU}$ ) and a single VMS resulting in ejection of the $8 \mathrm{M}_{\odot}$ star with a velocity from 200 to $700 \mathrm{~km} \mathrm{~s}^{-1}$ (top to bottom).
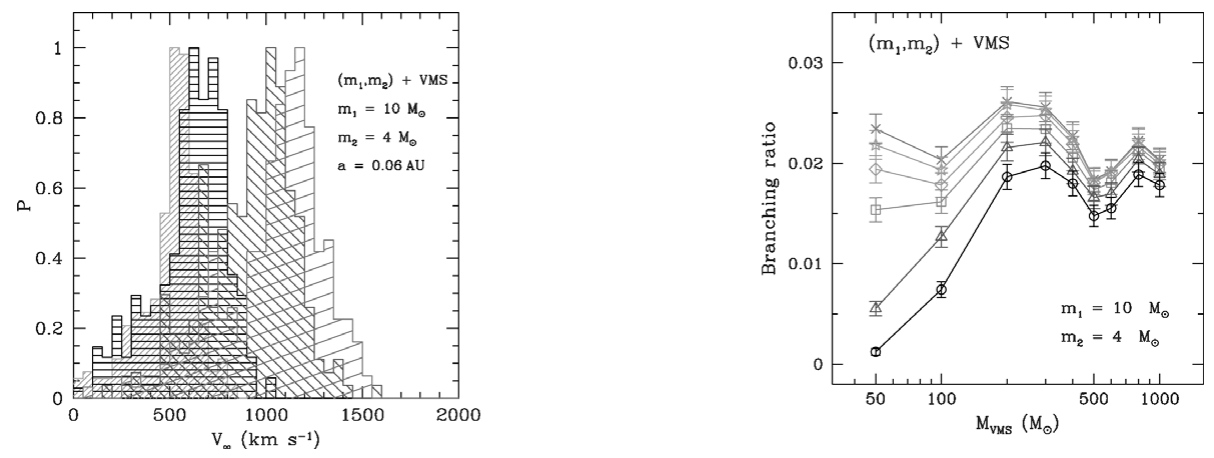

Figure 2. (left) Velocity distributions at infinity for escaping stars in encounters between a binary consisting of a primary star with mass $m_{1}=10 \mathrm{M}_{\odot}$ and a secondary star with mass $m_{2}=4 \mathrm{M}_{\odot}$, and a single VMS of mass $M_{\mathrm{VMS}}=50 \mathrm{M}_{\odot}, 100 \mathrm{M}_{\odot}, 500 \mathrm{M}_{\odot}$ and $1000 \mathrm{M}_{\odot}$ (from left to right). The binary semi-major axis is $a=0.06 \mathrm{AU}$. (right) Probability of exchange encounters between a $(10,4) \mathrm{M}_{\odot}$ binary (with $a=0.06 \mathrm{AU}$ ) and a single VMS resulting in ejection of the $4 \mathrm{M}_{\odot}$ star with a velocity from 200 to $700 \mathrm{~km} \mathrm{~s}^{-1}$ (top to bottom).

escapers. As expected, the more massive VMSs are more likely to eject stars with high velocities. In the right panel of Figure 1 we show the probability of exchange encounters resulting in ejection of the $8 \mathrm{M}_{\odot}$ binary component with a velocity from 200 to $700 \mathrm{~km} \mathrm{~s}^{-1}$ (top to bottom). For $M_{\mathrm{VMS}} \geqslant 100 \mathrm{M}_{\odot}$, about $3 \%$ of all encounters produce runaways with peculiar velocities $\geqslant 400 \mathrm{~km} \mathrm{~s}^{-1}$. It can be seen that even an ordinary star of mass of $50 \mathrm{M}_{\odot}$ can occasionally (in $\sim 1 \%$ of encounters) produce an escape velocity $\geqslant 400 \mathrm{kms}^{-1}$. To produce escapers with velocities typical of HVSs $\left(V_{\infty} \geqslant 700 \mathrm{~km} \mathrm{~s}^{-1}\right)$, a VMS of several hundred solar masses is required. For a $200-300 \mathrm{M}_{\odot}$ VMS, $\geqslant 2 \%$ of all encounters result in an escape velocity of $\geqslant 700 \mathrm{~km} \mathrm{~s}^{-1}$. This fraction gradually increases to $\geqslant 3 \%$ for the more massive VMSs.

Next, we consider exchange encounters producing high-velocity late B-type stars and simulate encounters between a very tight binary $\left(m_{1}=10 \mathrm{M}_{\odot}, \mathrm{m}_{2}=4 \mathrm{M}_{\odot}\right.$ and $a \simeq$ $\left.3 r_{1} \simeq 0.06 \mathrm{AU}\right)$ and a (very) massive star of mass of $50-1000 \mathrm{M}_{\odot}$. The velocity distributions for $4 \mathrm{M}_{\odot}$ escapers are shown in Figure 2 (left panel) for four different values of the VMS mass: $M_{\mathrm{VMS}}=50,100,500$ and $1000 \mathrm{M}_{\odot}$. The right-hand panel of Figure 2 
shows that for all values of $M_{\mathrm{VMS}}$ about $2 \%$ of encounters result in peculiar velocities $\geqslant 300-400 \mathrm{~km} \mathrm{~s}^{-1}$ and that about the same percentage of ejected stars attains velocities $\geqslant 700 \mathrm{~km} \mathrm{~s}^{-1}$ if $M_{\mathrm{VMS}} \geqslant 200 \mathrm{M}_{\odot}$.

We therefore argue that the origin of high-velocity $\left(\geqslant 200-400 \mathrm{~km} \mathrm{~s}^{-1}\right)$ early and late B-type runaway stars and at least some HVSs could be associated with dynamical encounters between the tightest massive binaries and VMSs formed in the cores of YMSCs. Future proper-motion measurements of HVSs with Gaia will reveal what fraction of these extremely-high-velocity stars originated in the Galactic disk.

\section{Acknowledgements}

We are grateful to L. R. Yungelson for useful discussions. VVG acknowledges the Russian Foundation for Basic Research and the International Astronomical Union for travel grants and the Deutsche Forschungsgemeinschaft for partial financial support. AG is supported by NASA grant NNX07AH15G. SPZ acknowledges support from the Netherlands Organization for Scientific Research (NWO; grant 643.200.503) and the Netherlands Research School for Astronomy (NOVA).

\section{References}

Baumgardt, H., Gualandris, A., \& Portegies Zwart, S. 2006, MNRAS, 372, 174

Belkus, H., Van Bever, J., \& Vanbeveren, D. 2007, ApJ, 659, 1576

Blaauw, A. 1961, Bull. Astron. Inst. Neth., 15, 265

Brown, W. R., Geller, M. J., Kenyon, S. J., \& Kurtz, M. J. 2005, ApJ (Letters), 622, L33

Chatterjee, S., Vlemmings, W. H. T., Brisken, W. F., Lazio, T. J. W., Cordes, J. M., Goss, W. M., Thorsett, S. E., Fomalont, E. B., Lyne, A. G., \& Kramer, M. 2005, ApJ (Letters), 630, L61

Gualandris, A. \& Portegies Zwart, S. 2007, MNRAS (Letters), 376, L29

Gualandris, A., Portegies Zwart, S., \& Sipior, M. S. 2005, MNRAS, 363, 223

Gvaramadze, V. V. 2007, A\&A (Letters), 470, L9

Gvaramadze, V. V. 2009, MNRAS (Letters), 395, L85

Gvaramadze, V. V., Gualandris, A., \& Portegies Zwart, S. 2008, MNRAS, 385, 929

Gvaramadze, V. V., Gualandris, A., \& Portegies Zwart, S. 2009, MNRAS, 396, 570

Heber, U., Edelmann, H., Napiwotzki, R., Altmann, M., \& Scholz, R.-D. 2008, A $\mathscr{G} A$ (Letters), 483, L21

Hills, J. G. 1988, Nature, 331, 687

Leonard, P. J. T. 1991, AJ, 101, 562

McMillan, S. L. W. \& Hut P. 1996, ApJ, 467, 348

Portegies Zwart, S. F. 2000, ApJ, 544, 437

Portegies Zwart, S. F. \& McMillan, S. L. W. 2002, ApJ, 576, 899

Portegies Zwart, S. F., McMillan, S. L. W., Hut, P., \& Makino, J. 2001, MNRAS, 321, 199

Poveda, A., Ruiz, J., \& Allen, C. 1967, Bol. Obs. Tonantzintla Tacubaya, 4, 86

Weidner, C., Kroupa, P., \& Bonnell, I. 2009, MNRAS, in press (arXiv:0909.1555)

Yu, Q. \& Tremaine, S. 2003, ApJ, 599, 1129

Yungelson, L. R., van den Heuvel, E. P. J., Vink, J. S., Portegies Zwart, S. F., \& de Koter, A. 2008, A\&BA, 477, 223 